\title{
Acompañando el Fortalecimiento de Capacidades Organizativas con Emprendedores y Emprendedoras Comunitarios: Lineamientos Metodológicos
}

\author{
Karla Córdoba Brenes ${ }^{1}$ \\ Jilma Pereyra López²
}

\section{RESUMEN}

Partiendo de la investigación Construyendojuntos/as nuestra realidad: Propuesta de Lineamientos Metodológicos de Acompañamiento para Procesos de Fortalecimiento de Capacidades Organizativas con Emprendedores y Emprendedoras Comunitarios se presenta en este artículo el resultado del análisis documental, del Estudio de Caso y del comparativo de experiencias, desde los cuales se teoriza y se proponen Lineamientos Metodológicos de Acompañamiento, guías generales para el planeamiento y diseño de este tipo de procesos. El análisis documental permite plantear una sólida fundamentación teórica, conceptual y metodológica para el desarrollo de la investigación. Por otra parte, el Estudio de Caso permite profundizar en el proceso en estudio, su contexto, sus aciertos y desaciertos. El análisis comparativo aporta perspectiva y contexto. Se busca así, rescatar las mejores experiencias para el planteamiento de posibles escenarios futuros de implementación de procesos similares. Estos escenarios parten de la clasificación de los procesos en aquellos con Enfoque en Negocios (EN) y aquellos con Enfoque en Capacidades Organizativas (ECO), y propone que la combinación de estos elementos debe ser intencionada puesto que influye en el fortalecimiento de capacidades para la implementación de Estrategias Asociativas (EA) por parte de emprendedoras y emprendedores comunitarios.

Palabras Clave: Lineamientos Metodológicos, Acompañamiento, Emprendedurismo, Capacidades Organizativas, Estrategias Asociativas.

Recibido: 10 de diciembre de 2014

Aceptado: 17 de marzo de 2015

1 Comunicadora Social especialista en Desarrollo Comunitario con experiencia en el sector ambiental y ONG, facilitadora de procesos comunales relacionados con empoderamiento, conservación y sostenibilidad. Correo Electrónico:muguika@gmail.com

2 Maestra en Psicología, atención personalizada y Desarrollo Social/Especialista en Gestión del Desarrollo Comunitario /Psicóloga/ Docente en FAREM Estelí, UNAN Managua/Consultora independiente. Correo Electrónico: jplo42@yahoo.com 


\title{
Accompanying the Strengthening of Organizational Capacity with Entrepreneurs and Community Entrepreneurs: Methodological Guidelines
}

\begin{abstract}
Based on the research Building Together our Reality: Proposed Methodological Guidelines of Accompanying for Strengthening Processes of Organizational Capacity with Entrepreneurs and Community Entrepreneurs it is presented in this article the results of the documented analysis, the case study, and the comparative experiences, from which it is theorized and Methodological Guidelines of Accompanying are proposed, general guidelines for planning and designing these processes. The documented analysis allows proposing a sound theoretical, conceptual, and methodological foundation for the development of the research. Furthermore, the case study gives insight into the process under study, its context, its successes and its failures. The comparative analysis provides perspective and context. It seeks therefore, to rescue the best experiences for the planning of possible future scenarios for the implementation of similar processes. These scenarios are based on the classification of processes on those with a Focus on Business (EN) and those with Focus on Organizational Capabilities (ECO), and it suggested that the combination of these elements must be intentional since it influences the capacity building for Associative Implementation Strategies (EA) by entrepreneurs and community entrepreneurs.
\end{abstract}

Keywords: Methodological Guidelines, Accompanying, Entrepreneurship, Organizational Skills, Associative Strategies. 
Construyendo Juntos/as Nuestra Realidad... una introducción

Las y los emprendedores locales son actores/as clave en el desarrollo de sus comunidades. En la búsqueda del empoderamiento y la autogestión, diseñan e implementan proyectos productivos que contribuyen a su bienestar individual, el de sus familias y el de sus comunidades, aunque no siempre cuentan con las capacidades organizativas necesarias para unirse a otras personas con espíritu emprendedor y facilitar su desarrollo conjunto.

Como facilitadora diseñé y acompañé el proceso Construyendo juntos nuestra propia realidad: Fortalecimiento Organizacional para Emprendedores en Turismo Rural Comunitario, desarrollado en la Península de Osa (sur de la provincia de Puntarenas, Costa Rica), entre 2012 y 2013, como parte del proyecto Promoción del Turismo Rural Comunitario para la Conservación de los Bosques en el Área de Conservación Osa, cuyo objetivo era contribuir con la autosostenibilidad de las comunidades locales, su empoderamiento y la conservación de un valioso recurso: sus bosques (Guindaleza, 2013). Como resultado de este proceso las y los emprendedores/ as decidieron organizarse bajo la modalidad de cooperativa, creando la Cooperativa de Emprendedores en Turismo Rural de Corcovado (COOPETURIC R.L.).

La Península de Osa es, según la revista National Geographic "el lugar más biológicamente intenso del mundo"*1. El contraste entre su riqueza natural y el bajo índice de Desarrollo Social que viven sus comunidades es un claro ejemplo de injusticia socioambiental.

En los últimos años, el turismo se posicionó como fuente complementaria de ingresos para las comunidades, aunque no todos los modelos de turismo resultan idóneos para esta ecológicamente frágil región.

1 http://en.wikipedia.org/wiki/Corcovado_National_Park
Un modelo de turismo rural comunitario (TIC) representa una alternativa importante, disminuyendo la presión sobre los bosques y estimulando la sensibilización hacia la conservación (Neotrópica, 2010). En total, los 11 emprendimientos que conforman COOPETURIC conservan más de 900 hectáreas de bosque tropical lluvioso (González, 2013).

Como facilitadora reconozco que el proceso tuvo sus aciertos y desaciertos, de ahí el deseo de contrastar la experiencia con las propuestas de otros procesos de formación similares y de formular una Propuesta de Lineamientos Metodológicos de Acompañamiento que sea implementada con esas personas y familias que requieren fortalecer sus capacidades organizativas, reconstruir su realidad y al mismo tiempo, conservar los valiosos recursos naturales con los que cuentan.

\section{Construyendo Juntos/as... desde la Metodología}

Construimos entonces desde un paradigma interpretativo y humanista, buscando comprender la dinámica de las y los emprendedores comunitarios, los retos a los que se enfrentan frente a la organización comunitaria y las capacidades que podrían ayudarles a enfrentar de una mejor manera estos retos. Se parte de que su realidad es cambiante y se trabaja con base en un modelo dialéctico, donde el conocimiento es resultado de un diálogo entre el sujeto (sus intereses, valores, creencias, etc.) y el objeto de estudio (Martínez, 2006). La investigación se desarrolla con plena conciencia de que como facilitadora, su autora es sujeta partícipe en la construcción de la historia, totalmente implicada de forma activa en su proceso (Jara, s.f.).

Paso 1 | Establecimiento de objetivos, objeto de estudio $\mathrm{y}$ alcances.

Paso 2 | Revisión documental y síntesis de perspectivas teóricas de investigadores como Van de Velde (2008, 2012), Jara (s.f.) y Martinez (2006), desde un paradigma interpretativo y humanista, considerando 
los principales postulados de la educación popular, la concepción metodológica dialéctica, la dimensión política del trabajo con grupos comunitarios, la metodología P-COA_acem (Procesos de Construcción Conjunta de Oportunidades de Aprendizaje, integrante de una actitud emprendedora de calidad) y la pedagogía del SER. Se definieron conceptos clave como organización comunitaria, autogestión, autonomía, empoderamiento, emprendedurismo comunitario, procesos participativos y capacidades organizativas, además de un breve repaso de la cooperativa como opción organizativa y productiva.

Paso 3 | Desarrollo del Estudio de Caso. Se planteó un Estudio de Caso Simple (Yin, 2009), reconociendo que su selección es motivada tanto por lo que tiene de único como por lo que tiene de común (Stake, 1998). Se eligió el caso por la relación directa de la investigadora con el proceso inicial y por la relevancia del caso en la zona y en el proceso de conformación de COOPETURIC, que eligió la cooperativa como Estrategia Asociativa (EA). El estudio de caso se construyó a partir de los documentos del proyecto, los informes de proceso (IdP) y las entrevistas a profundidad realizadas a cuatro miembros de COOPETURIC (2 mujeres y 2 hombres) participantes del proceso.

Paso 4 Análisis comparativo de experiencias de formación similares. Este tipo de procesos incluyen contenido con Enfoque en Negocios (EN) y con Enfoque en Capacidades Organizativas (ECO). El primer enfoque $(\mathrm{EN})$ se refiere a la formación técnica en el área de negocios, conocido también como enfoque en "competencias" técnicas. El segundo enfoque (ECO) se centra en las capacidades para fortalecer el SER y trabajar en equipo como una organización. Este enfoque también es conocido como enfoque en desarrollo humano o actitudes/habilidades personales. El análisis comparativo consideró la distribución de módulos, el contenido de cada módulo, el análisis de enfoque (EN o ECO) de cada contenido y el análisis acumulado de enfoque (EN o ECO) por módulo. Se seleccionaron cinco experiencias implementadas en América Central y América del Sur.

Paso 5 | Desarrollo de la Propuesta de Lineamientos Metodológicos de Acompañamiento.

Construyendo Juntos/as... y contemplando los alcances y limitaciones de la investigación

Como en todos los procesos sociales, no existen recetas ni paso-a-paso infalibles a seguir. Los lineamientos metodológicos de acompañamiento se configuran solamente como luces en el camino.

El Estudio de Caso se estructura y se analiza desde la experiencia de la facilitadora (autora de esta investigación) y gracias a los insumos y aportes de los/as emprendedores/as entrevistados/as. No fue posible incluir los sentires y pensares de todos y todas los emprendedores, aunque una inclusión de mayor cantidad de puntos de vista podría ayudar a configurar más en detalle los lineamientos propuestos, o bien, a validarlos.

No pretende esta investigación mostrar un análisis exhaustivo de experiencias, sino destacar las que la autora considera relevantes según los criterios expuestos. Existen muchas y ciertamente interesantes experiencias que pueden ser recopiladas, analizadas y convertidas en lineamientos para el trabajo, en otras áreas y contextos.

\section{Construyendo Juntos/as... sobre el Estudio de Caso y otras experiencias latinoamericanas similares}

Según se cita en el informe final del proyecto (González, 2013) su objetivo era "facilitar la conservación de áreas boscosas a través de la creación de una red de turismo rural comunitario, como medio para solventar la carencia de actividades productivas sostenibles en el Área de Conservación Osa”. Las y los participantes del proyecto, miembros/as hoy de COOPETURIC, habitan 
el cantón de Osa y tienen en común un sueño: asegurar el bienestar de sus familias, de sus comunidades y de su entorno ambiental. Este estudio de caso se centra en la experiencia vivenciada por 18 miembros de COOPETURIC, quienes participaron del proceso. El $100 \%$ de los emprendimientos están conformados por familias locales.
El Proyecto se planteó una estrategia de ejecución con base en 2 grandes bloques, 4 etapas, 5 objetivos específicos y sus correspondientes actividades, según se detalla en el cuadro a continuación.

\begin{tabular}{|c|c|c|c|c|}
\hline Bloque & Etapa & Objetivos Específicos & Actividades & Comentarios \\
\hline \multirow{3}{*}{$\begin{array}{l}\text { Bloque } 1 \\
\text { Fortalecimiento } \\
\text { de negocios } \\
\text { (individual) }\end{array}$} & \multirow[t]{2}{*}{$\begin{array}{l}\text { Etapa } 1 \\
\text { Entrenamiento }\end{array}$} & $\begin{array}{l}\text { Objetivo } 1 \\
\text { Identificar al menos } \\
\text { un proyecto turístico } \\
\text { en cada una de las } \\
12 \text { comunidades } \\
\text { involucradas en el } \\
\text { proceso }\end{array}$ & Proceso de selección & $\begin{array}{l}\text { Inicialmente } \\
\text { el proyecto } \\
\text { i8dentificó } 12 \\
\text { comunidades } \\
\text { de interés. Se } \\
\text { seleccionaron } 16 \\
\text { emprendimientos } \\
\text { de } 10 \\
\text { comunidades. }\end{array}$ \\
\hline & & $\begin{array}{l}\text { Objetivo } 2 \\
\text { Capacitar miembros } \\
\text { de las comunidades } \\
\text { para operar proyectos } \\
\text { de TRC y actividades } \\
\text { complementarias }\end{array}$ & $\begin{array}{l}3 \text { jornadas de capacitación } \\
\text { de } 3 \text { días cada un Manual } \\
\text { de Buenas Prácticas para } \\
\text { TRC. Capacitación en } \\
\text { elaboración de artesanías } \\
\text { de alta calidad a partir de } \\
\text { materiales reciclados }\end{array}$ & $\begin{array}{l}\text { Permanecen en } \\
\text { el proceso } 11 \\
\text { emprendimientos } \\
\text { de } 6 \text { comunidades, } \\
\text { quienes } \\
\text { conformaron } \\
\text { COOPETURIC. } \\
\end{array}$ \\
\hline & $\begin{array}{l}\text { Etapa } 2 \\
\text { Implementación }\end{array}$ & $\begin{array}{l}\text { Objetivo } 3 \\
\text { Iniciar proceso de } \\
\text { Implementación } \\
\text { sostenible de } \\
\text { proyectos de TRC y } \\
\text { complementarios }\end{array}$ & $\begin{array}{l}\text { Planes de negocio, fuentes } \\
\text { de financiamiento } \\
\text { Acompañamiento y } \\
\text { asesoría. }\end{array}$ & $\begin{array}{l}\text { Permitió } \\
\text { consolidación de } \\
\text { ideas de negocio y } \\
\text { fortalecimiento de } \\
\text { emprendimientos } \\
\text { en } \\
\text { funcionamiento. } \\
\text { Se contó con } 11 \\
\text { emprendimientos } \\
\text { cada uno con su } \\
\text { plan de negocios. }\end{array}$ \\
\hline
\end{tabular}


Bloque 2

Fortalecimiento

de red

(colectivo)
Objetivo 4

Etapa 3

Integración

comunal
Formar una estructura

organizativa

(cooperativa $\mathrm{u}$

asociación) para

impulsar el TRC
Intercambio de

experiencias a lo interno

Intercambio de

experiencias con

asociaciones y

cooperativas con

trayectoria en TRC.

Proceso de

fortalecimiento en temas

de autoestima, capacidad

organizativa y relaciones

humanas.

Asesoría para analizar las

figuras de asociación y

cooperativa

Elección de figura y

formación de cooperativa

(nombre legal y

comercial)

Inscripción legal

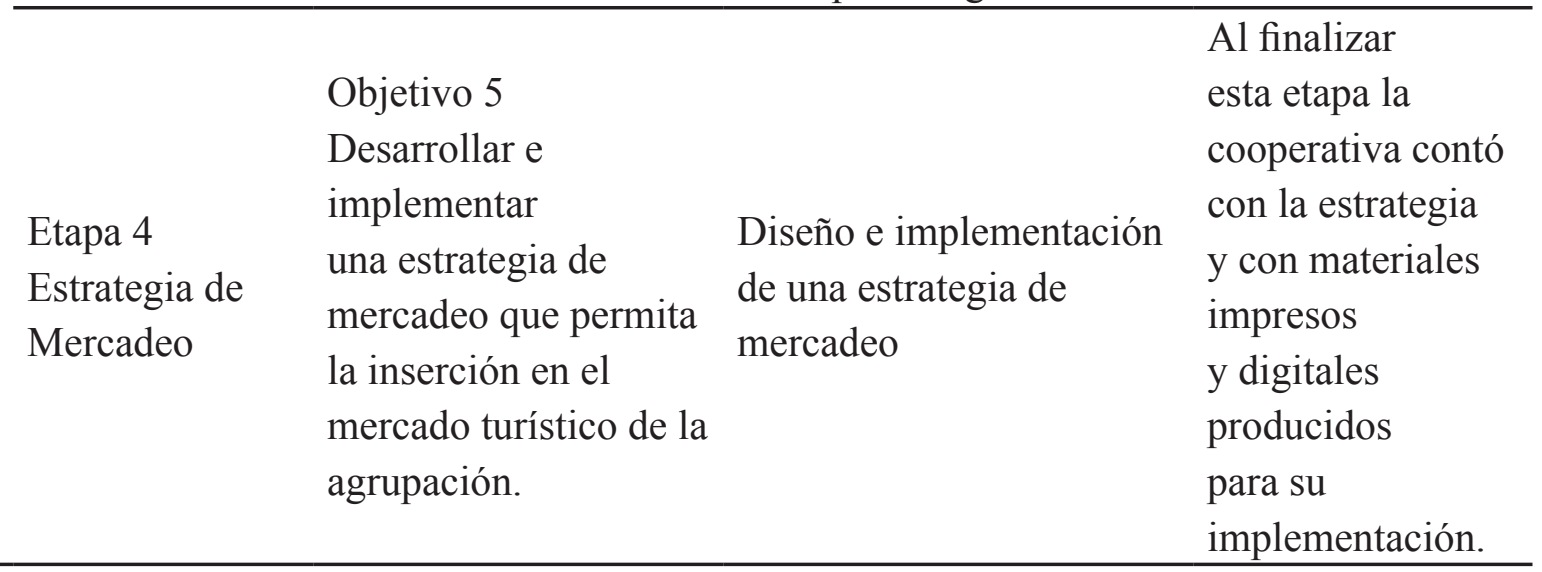

Cuadro 01. Estructura del proyecto. (Elaboración propia con base en González, 2013)

El proceso "Construyendo Juntos Desde Nuestra Propia Realidad: Fortalecimiento Organizacional para Emprendedores de Turismo Rural Comunitario" contribuyó al cumplimiento del objetivo 4, etapa 3 del bloque 2, y es mencionado como "Proceso de fortalecimiento en temas de autoestima, capacidad organizativa y relaciones humanas". Su objetivo fue "facilitar un proceso en el que las y los emprendedores de TRC desarrollen valores y capacidades que les permitan trabajar en red $y$ desarrollarse organizativamente tomando en cuenta su propia realidad y la de su emprendimiento" (Córdoba, 2012). Estos talleres se convierten en dispositivos para la autogestión que permiten la formación de los socios potenciales de la cooperativa (Heras, 2011), la reflexión sobre la experiencia y la facilitación de un espacio de construcción de conocimientos en torno a la autonomía y e empoderamiento desde un enfoque de emprendedurismo comunitario. 
Se estructuró como un proceso en que las y los emprendedores serían acompañados más que dirigidos. Se define el rol de los/as encargados/as como "facilitadores", se valora el intercambio de saberes y sentires, y estos están conscientes de su rol como acompañantes del aprendizaje y del desarrollo.

El Enfoque Metodológico del proceso planteó una metodología participativa y experiencial o vivencial,

\begin{tabular}{|c|c|c|c|}
\hline Taller & Tema & Título & Descripción \\
\hline 1 & Autoestima & $\begin{array}{l}\text { ¿Quién soy?: mi historia y la de mi } \\
\text { emprendimiento. Construyendo a partir } \\
\text { de lo que existe }\end{array}$ & $\begin{array}{l}\text { Sesión enfocada en el emprendedor, su } \\
\text { vida, valores y prioridades, la historia } \\
\text { de su emprendimiento, sus fortalezas y } \\
\text { oportunidades. }\end{array}$ \\
\hline 2 & $\begin{array}{l}\text { Capacidad } \\
\text { Organizativa }\end{array}$ & $\begin{array}{l}\text { ¿Quiénes somos como colectivo?: } \\
\text { Nuestras historias se entrelazan. } \\
\text { Construyendo a partir de nuestras } \\
\text { individualidades }\end{array}$ & $\begin{array}{l}\text { Sesión enfocada en el grupo como } \\
\text { colectivo, valores, fortalezas, } \\
\text { oportunidades .Se trabajaría bajo los } \\
\text { principios de la planificación, construyendo } \\
\text { juntos la organización. }\end{array}$ \\
\hline 3 & $\begin{array}{l}\text { Relaciones } \\
\text { Humanas }\end{array}$ & $\begin{array}{l}\text { ¿Cómo nos relacionamos?: } \\
\text { Comunicación, Conflictos y Liderazgo. } \\
\text { Construyendo a partir de nuestras } \\
\text { interacciones }\end{array}$ & $\begin{array}{l}\text { Sesión enfocada en trabajo en equipo y } \\
\text { logro de objetivos comunes. }\end{array}$ \\
\hline
\end{tabular}

Cuadro 02. Propuesta de Talleres y Temas del Proceso (Córdoba, 2012).

Es común encontrar procesos de formación para emprendedores con una amplia gama de contenidos con EN. Lo que no es tan común, es encontrar procesos que además de estas habilidades, promuevan el desarrollo de capacidades organizativas en estos grupos de emprendedores/as.

Un facilitador o facilitadora con el reto de desarrollar los contenidos y el diseño metodológico para procesos del segundo tipo, se encontrará fácilmente con referencias inspirada en las propuestas del pedagogo brasileño Paulo Freire (Córdoba, 2012). El proceso buscaba "el aprendizaje a través de la propia realidad, en un lenguaje sencillo y aplicando la horizontalidad facilitador-participante" y se estructuró como una etapa previa a la consolidación del grupo como un ente colectivo con una misión y una visión común.

y ejemplos de procesos con EN. Partir solamente de estas referencias puede redundar en nuevos procesos que no contemplen las capacidades organizativas, en detrimento del proceso de las y los emprendedores que buscan trabajar como colectivo.

Sin ningún afán exhaustivo se seleccionaron cinco propuestas latinoamericanas (detalladas a continuación) que integran el ECO, para desarrollar un análisis de su estructura. 
Proceso

Región Centroamericana: Belice,

Reto Emprendedor

Guatemala, El Salvador, Honduras,

País/Región Nicaragua, Costa Rica, Panamá, República Dominicana

Organicemos las ideas Manual para Emprendedores Turísticos Estrategias Empresariales para jóvenes Emprendedores

Capacitación en Gestión Social y Emprendedurismo

Argentina

Costa Rica
Organización gestora

Centro para la Promoción de la Micro y Pequeña Empresa en Centroamérica (CENPROMYPE), Sistema de Integración Centroamericana (SICA)

Secretaría de Turismo

Instituto Nacional de Aprendizaje (INA)

Organización de las Naciones

Unidas para la Alimentación y la Agricultura (FAO)

Asociación Incubadora Parque TEC

Curso de Capacitac
Gestión Empresarial

Costa Rica

Cuadro 03. Propuestas seleccionadas para el análisis comparativo de procesos de formación con emprendedores y emprendedoras. Elaboración propia.

Se procedió a sistematizar los principales contenidos agrupados por módulos y se asignó una clasificación a cada módulo según el enfoque del contenido: EN o ECO. Se incluye el siguiente diagrama, en el que las experiencias están identificadas como P1, P2, P3, P4 y P5; los módulos están identificados por círculos; los módulos correspondientes a ECO se identifican con las siglas dentro del círculo correspondiente; se distribuyen los módulos en dos bloques (Bloque 1 y Bloque 2).

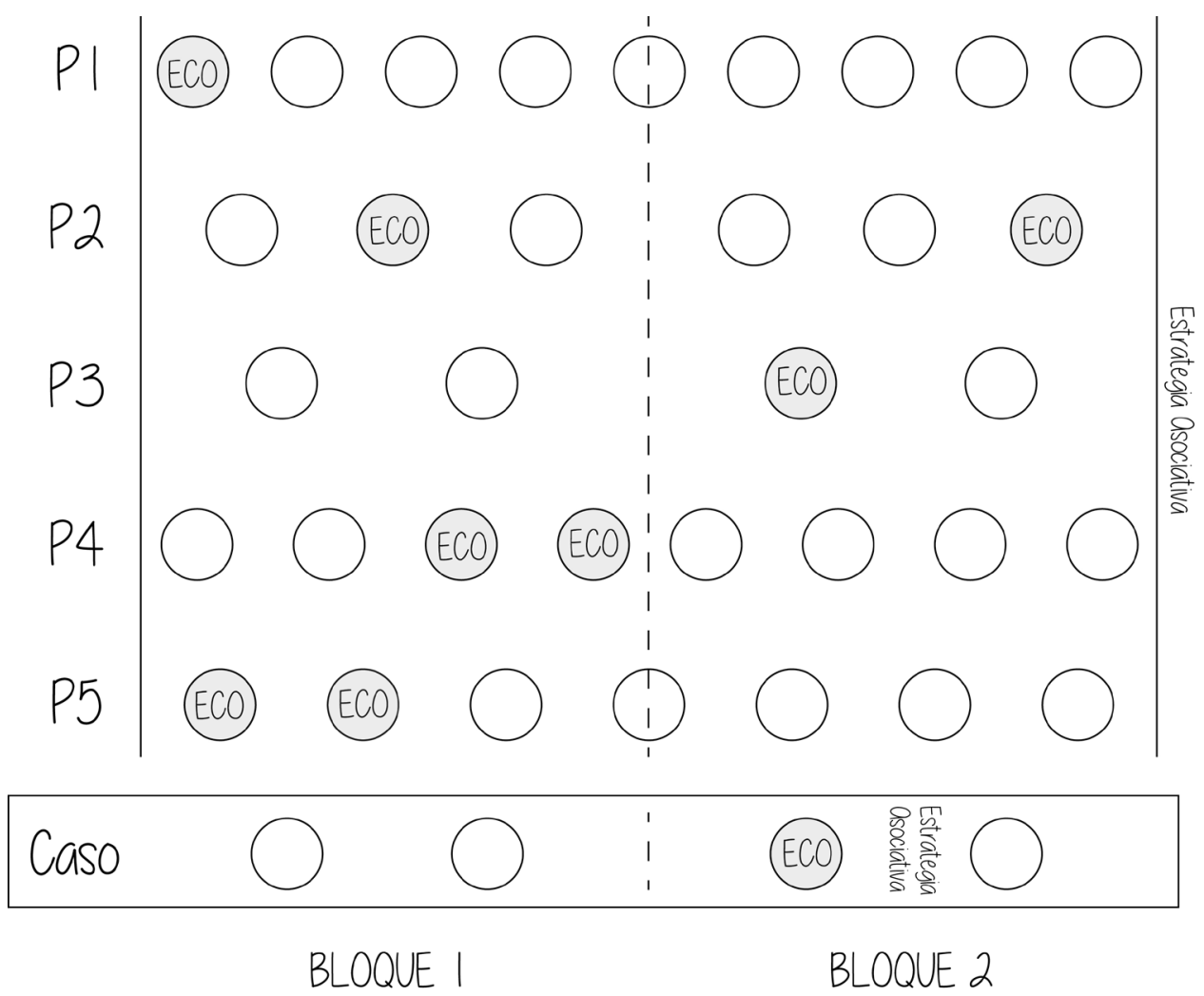

Imagen 01. Diagrama comparativo de la estructura de las experiencias según el enfoque de sus módulos, incluyendo el Estudio de Caso. Elaboración propia. 
El diagrama incluye la información del Estudio de Caso y la referencia al momento en que se implementa la EA (texto vertical). Para nuestro Estudio de Caso la EA seleccionada fue la conformación de la cooperativa, la cual se implementó inmediatamente después del proceso de formación con ECO. Para las demás experiencias, la EA se implementa al final, una vez concluido el proceso de formación.

\section{Construyendo Juntos/as... lo que aprendimos}

Las y los emprendedores entrevistados afirmaron que el proceso contribuyó a la formación del grupo de emprendedores/as y su consolidación como COOPETURIC. Anteriormente al proceso se encontraban desarticulados.

Valoraron positivamente temas relacionados con Autoestima, Identidad, Valores y Capacidades Organizativas generales. Señalaron la importancia de fortalecer el liderazgo, manejo de conflictos y trabajo colaborativo. Evaluaron positivamente la metodología, la empatía, la apertura y el uso de recursos de recreación con propósito. Recomendaron que algunos de los temas se abordarían mejor a nivel individual o con apoyo de un/a consejero/a o psicólogo/a.

Resaltaron que las personas presentan motivaciones y agendas diferentes en cuanto a por qué participar, por lo que el enfoque metodológico es fundamental. Considerando lo anterior, durante el proceso se aplicó la Regla de 3, para promover la participación tanto individual, como en grupos pequeños y en plenaria.

El cambio entre un enfoque $(\mathrm{EN})$ y otro $(\mathrm{ECO})$ durante el proceso no fue percibido con tanta claridad por las y los emprendedores. No coincidieron en una estructura ideal entre ECO y EN, sino que hicieron énfasis en que cada caso es particular. Resalta la importancia de que la formación con ECO se dé cercana a la implementación de la EA.
Integrar la perspectiva de los emprendimientos como negocios familiares y las estrategias para manejar sus relaciones y conflictos es fundamental, incluyendo temas como autoridad y poder.

Una de las principales recomendaciones es integrar a las y los emprendedores en la facilitación y búsqueda de información y formulación de la metodología.

Desde la perspectiva de la facilitadora el proceso propició el descubrimiento de cualidades propias y cualidades del otro, así como la importancia de las historias personales y de los emprendimientos. Al no contar con una línea base con el perfil de cada uno/una, se dificultó la identificación de conflictos previos entre los participantes y la evaluación.

No siempre fue posible cumplir con los diseños metodológicos de las actividades ni se contó con una evaluación participativa al finalizar. Es necesario reforzar el cierre de cada actividad identificando las alternativas de solución y aplicando con ejemplos concretos lo aprendido o descubierto durante la sesión.

En cuanto al análisis comparativo de experiencias, todas las iniciativas incluyen las potenciales EA después del proceso formativo. Ninguna de las experiencias en estudio incluyó la formación de una organización nueva durante el proceso, a excepción del Estudio de Caso.

Del total de módulos de las iniciativas, solamente $25 \%$ corresponde a contenidos con ECO. Esta es la misma proporción presente en nuestro Estudio de Caso. Se nota una predominancia de ECO hacia la primera parte del proceso (Bloque 1). La ubicación de contenidos con ECO se encuentra mayormente alejada de la implementación de las EA. Solamente una de las iniciativas (P2), además de nuestro Estudio de Caso, incluye contenidos con ECO al final del proceso de formación. 
Construyendo Juntos/as... la Propuesta de Lineamientos.

El mejor momento para fortalecer capacidades organizativas: ;antes de organizarse!: Cuando trabajamos con un grupo de emprendedores/as comunitarios que están buscando organizarse, es el momento óptimo para fortalecer estas capacidades.

Estableciendo los objetivos: Claridad en cuanto a las expectativas de lasylos emprendedores ylasnecesidades inmediatas y futuras de sus emprendimientos.

Perfil previo: Contiene información básica personal (datos de contacto, condiciones de aprendizaje, experiencia, actividades productivas, etc.), familiar (núcleo, interacciones, historia, economía, cultura, etc.), de participación en procesos similares (experiencias positivas y negativas) y expectativas del proceso. Se recomienda crearlo a partir de entrevistas personales, familiares, tests y observación, a manera de línea base.

Facilitación: E1/la facilitador/a deberá conocer a profundidad el grupo, propiciar el diálogo y la reflexión en un ambiente de respeto mutuo y fraternidad, cuidar los tiempos y estructuras metodológicas.

Dimensiones: Integrar la dimensión individual (autointerrogación, potenciación de la propia vitalidad, lo que me hace persona, no solo emprendedor) y la colectiva (mi relación con el/la otro/a, relaciones y responsabilidades compartidas como compañeros/as y emprendedores/as) en todas las actividades.
Recomendaciones Metodológicas: Se recomienda una estructura basada en talleres, con mezcla entre actividades teóricas y prácticas; aplicación de elementos lúdicos que propicien momentos significativos; análisis DÍA (Descripción-Interpretación-Aplicación) como herramienta de análisis post actividad; cuido de tiempos máximos y mínimos; calidad y cantidad de materiales y espacios físicos; organización del grupo en círculo (contacto visual y mismo nivel de conversación); inclusión de invitados del núcleo familiar, inclusión de asignaciones para el hogar que se comparten posteriormente; aplicación de evaluación participativa por sesión; construcción de Memorias Significativas y Momentos Significativos en espacios libres; utilización de elementos simbólicos para la unión del grupo (distintivos, signos, fotografías); propiciar el intercambio de ideas y la escucha del otro/a; alternancia de trabajo individual, en grupos pequeños y plenaria (Regla de 3); refrescamiento constante del proceso y los objetivos; aplicación del modelo ULI (Unidad Local de Implementación) en la que se define un grupo de participantes encargado de apoyar la facilitación; y la inclusión de un llamado a la acción y alternativas concretas de aplicación al cierre de cada sesión.

Escenarios: Se plantean cuatro escenarios base. La decisión final de la estructura dependerá de las características particulares de los/as emprendedores/as involucrados/as, sus expectativas y objetivos, así como la estrategia general de la propuesta.

\begin{tabular}{llll}
\hline Escenario & \multicolumn{1}{c}{ Estructura } & \multicolumn{1}{c}{ Descripción } & \multicolumn{1}{c}{ Consideraciones } \\
\hline & & & $\begin{array}{l}\text { Se recomienda para } \\
\text { emprendimientos incipientes o de } \\
\text { desarrollo bajo y que requieran una }\end{array}$ \\
Escenario 1 & $\mathrm{EN} \rightarrow \mathrm{ECO} \rightarrow \mathrm{EA}$ & $\begin{array}{l}\text { Inia con EN. Hacia el final } \\
\text { se introducen temas con ECO, } \\
\text { rumbo a la conformación de la } \\
\text { EA seleccionada. }\end{array}$ & $\begin{array}{l}\text { estructuración previa o bien sea } \\
\text { necesario desarrollar un proceso de } \\
\text { selección de los emprendimientos } \\
\end{array}$ \\
& & antes de implementar la EA
\end{tabular}


Escenario 2 ECO $\longrightarrow$ EN $\rightarrow$ EA

Escenario 4
Inicia con ECO. Una vez consolidado el grupo de emprendedores, se continúa con el fortalecimiento con EN, para culminar con la implementación de la EA seleccionada.

Se mezclan contenidos con $\begin{array}{ll}\text { Escenario } 3 & (\mathrm{EN}+\mathrm{ECO}) \longrightarrow(\mathrm{EN} \\ +\mathrm{ECO}) \longrightarrow \mathrm{EA}\end{array}$ EN y con ECO en proporción de $3(\mathrm{EN})$ a 1 (ECO ) en cada actividad

Variante del escenario 1. Inicia con breve refuerzo con ECO, especialmente relación emprendimiento/familia (podría suprimirse si se integra en contenidos con EN), para continuar con EN. Al finalizar el proceso se refuerza con ECO.
Se recomienda cuando el Perfil inicial alerte sobre situaciones particulares que necesiten mediación y conciliación para evitar un resultado negativo en la EA. Los emprendimientos presentan un desarrollo medio o medio-alto.

Se recomienda para emprendimientos de desarrollo medio y perfiles que denotan necesidades de mediación no urgentes pero sí relevantes.

Se recomienda como escenario ideal para situaciones con presencia mayoritaria de emprendimientos familiares, relaciones internas conflictivas, y desarrollo medio-bajo de los emprendimientos.

Cuadro 03. Escenarios para la estructura del proceso. Elaboración propia con base en González, 2013)

Se recomienda implementar las actividades con ECO antes de la EA, excepto para el Escenario 2, en el que por razones extraordinarias sea necesario enfocar esfuerzos iniciales en el fortalecimiento de capacidades organizativas, con base en un análisis detallado de los perfiles previos.

Contenidos sugeridos: Autoestima; Historia de Vida; Emprendimiento y Familia; Género; Compromiso mutuo y genuino; Unión en la diferencia; Valores personales y colectivos; Comunicación interpersonal, verbal, no verbal y organizacional;, Asertividad y Empatía; Liderazgo e Influencia; Liderazgos múltiples y compartidos; Inteligencias múltiples; Identificación y manejo de conflictos; Conflictos personales, de negocio y de organización; Diálogo, negociación y consenso; Estrategias Asociativas; y Aprendizaje Continuo.
Construyendo Juntos/as... Conclusiones, Lecciones Aprendidas y Recomendaciones

Los procesos de acompañamiento con emprendedores/ as comunitarios son un espacio poderoso para el cambio y la movilización social; ofrecen una ventana para comprender la dinámica de nuestras comunidades, a la luz de sus inquietudes productivas y de autogestión.

El proceso Construyendo Juntos Nuestra Propia Realidad fue un proceso participativo que facilitó la formalización y consolidación de COOPETURIC y permitió el fortalecimiento de las capacidades organizativas de los/as emprendedores/as. $\mathrm{La}$ metodología participativa y vivencial recibió valoraciones positivas, aunque presenta oportunidades de mejora. Si bien el proceso arrojó resultados positivos, está lejos de ser un proceso $100 \%$ replicable. Deberá ser revisado, contrastado y evaluado a profundidad. 
La propuesta de lineamientos parte de una experiencia concreta, en un contexto concreto. Es responsabilidad de las y los facilitadores conocer la comunidad, la realidad y objetivos del grupo con el que trabajan $\mathrm{y}$ ajustar y proponer a partir de sus experiencias $\mathrm{y}$ realidades.

Se recomienda validar la propuesta con emprendedores/ as participantes del proceso y externos, que puedan ofrecer una visión más completa de la propuesta, así como con expertos (a través del método Delphi).

Serecomienda implementar los lineamientos planteados en la presente investigación, de manera que sea posible evaluar la experiencia, proponer modificaciones de mejora y cerrar el ciclo práctica-teoría-práctica.

La implementación del análisis sobre nuestra propia práctica y el análisis comparativo nos permite encontrar puntos de ajuste, nuevos aprendizajes y abordajes. Esta constante observación hacia adentro y hacia afuera nos permite desarrollar procesos cada vez más honestos, ajustados a la realidad y necesidades de las comunidades con las que trabajamos.

\section{REFERENCIAS BIBLIOGRÁFICAS}

Córdoba, K. (2012), Propuesta Talleres Construyendo Juntos Desde Nuestra Propia Realidad, Fortalecimiento Organizacional para Emprendedores de Turismo Rural Comunitario. Proyecto Promoción del Turismo Rural Comunitario para la Conservación de los bosques en el Área de Conservación Osa, Fundación Neotrópica, San José, 8 pp.

Córdoba, $\mathrm{K}$ (2015). Construyendo juntos/as nuestra realidad: Propuesta de Lineamientos Metodológicos de Acompañamiento para Procesos de Fortalecimiento de Capacidades Organizativas con Emprendedores y Emprendedoras Comunitarios. Universidad Nacional Autónoma de Nicaragua, Facultad Multidisciplinaria Estelo y ABACOenRed, San José, 193 pp.

Fundación Neotrópica, Fundación Corcovado (2010), Propuesta Promoción del Turismo Rural Comunitario para la conservación de los bosques en el Área de Conservación Osa, San José, 30 pp.

González, A. (2013), Informe Final. Proyecto Promoción del Turismo Rural Comunitario para la Conservación de los Bosques en el Área de Conservación Osa. Fundación Neotrópica, San José, 20 pp.

Heras Monner Sans, A. (2011), Pensar la autonomía. Dispositivos y mecanismos en proyectos de autogestión. Intersecciones en Comunicación (Argentina), 5, 31-64.

Jara, Oscar. (s.f.), Concepción Metodológica Dialéctica. Alforja, San José, 17 pp.

Martínez, M. (2006), La investigación cualitativa (Síntesis Conceptual). Facultad de Psicología UNMSM, Revista IIPSI, 9(1), p. 123-146.

Stake, R. (1998), Investigación con Estudio de Casos. Ediciones Morata, $159 \mathrm{pp}$.

Van de Velde, H. (2008), Educación Popular: texto de referencia y consulta. CICAP / VOLENS Centroamérica, Estelí, 166 pp.

Van de Velde, H. (2012), ¿Por qué y para qué competir si cooperar es 'esencia humana'? ABACOenRed, Estelí, Nicaragua, 13 pp.

Van de Velde, H. (2012), SER: Una pedagogia concienciadora de poder compartido. ABACOenRed, Estelí, Nicaragua, 15 pp.

Yin, R.(2009), Investigación sobre Estudios de Caso: Diseño y Métodos. Segunda Edición, Applied Social Research Methods Series, Cuarta Edición, Sage Publications, Londres, 35 pp. 